\title{
Diagnóstico diferencial de hemitórax opaco en radiografía simple: Reporte de un Caso Clínico
}

\section{Differential diagnosis of opaque hemithorax on plain radiography: Report of a clinical case}

Pág. 28,35

Recibido: 28-02-2019

Aceptado: 27-05-2020

Dr. Renato Brenes Barrantes. Médico Cirujano Asistente, Especialista en Cirugía de Tórax. Hospital San Juan de Dios, Costa Rica. Dra. Claudia Orozco Cubero. Médico General, Costa Rica.

\section{Resumen}

Hemitórax opaco se define como una opacidad completa de uno de los hemitórax, en una radiografía simple. El diagnóstico diferencial se basa en la posición del mediastino: volumen del hemitórax aumentado, el mediastino se desvía contralateral al lado afectado (masas torácicas grandes y derrame pleural masivo); volumen disminuido, el mediastino se desvía hacia el lado afectado (neumonectomía, agenesia, atelectasias, aplasia e hipoplasia pulmonar); volumen normal, no hay desviación (neumonía que afecte la totalidad del pulmón, o carcinoma bronquial con atelectasias y derrame pleural). Caso clínico: Femenina, 70 años, con tos productiva, hemoptisis, disnea, y disminución de ruidos respiratorios en base izquierda. Radiografía de tórax con Radioopacidad total del hemitorax izquierdo. TC de tórax muestra atelectasia obstructiva subtotal izquierda, secundaria a masa en bronquio principal izquierdo, a $4 \mathrm{~cm}$ de la carina; confirmada con broncoscopía. Se realiza neumonectomía y biopsia, que se reporta como Adenocarcinoma invasor.

Discusión: principales diagnósticos diferenciales en este caso clínico: hemotórax (borramiento del ángulo costodiafragmático, opacificación parcial o completa del hemitórax); derrame pleural (ausencia de ruidos respiratorios, matidez a la percusión y frémito vocal táctil; opacidad homogénea con obliteración del ángulo costofrénico); atelectasia (opacidad completa del hemitórax con desviación del mediastino).

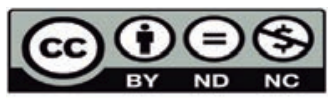

Conclusiones: El diagnóstico diferencial de un hemitórax opaco se basa en la posición del mediastino y el volumen de éste, en una radiografía simple. Las tres entidades más probables en la paciente del caso son: hemotórax, derrame pleural y atelectasias. La radioopacidad completa estaba asociada a una atelectasia, secundaria a Adenocarcinoma invasor.

\section{Abstract}

Opaque hemithorax is defined as a complete opacity of an hemithorax, in a simple radiography. The differential diagnosis is based on the position of the mediastinum: increased hemithorax volume, the mediastinum deviates contralaterally to the affected side (large thoracic masses and massive pleural effusion); decreased volume, the mediastinum deviates to the affected side (pneumonectomy, agenesis, atelectasis, aplasia and pulmonary hypoplasia); with normal volume, there is no deviation (pneumonia that affects the entire lung, or bronchial carcinoma with atelectasis and pleural effusion). Case report: Female, 70 years old, with productive cough, hemoptysis, dyspnea, and decreased respiratory sounds in the left base. Total radiopacity of the left hemithorax is seen in the chest $\mathrm{x}$-ray. Chest CT scan shows obstructive atelectasis, secondary to mass in the left main bronchus, $4 \mathrm{~cm}$ from the carina; which is confirmed with a bronchoscopy. Pneumonectomy is performed and a biopsy is taken (reported as invasive adenocarcinoma). 
Discussion: main differential diagnoses in this clinical case: hemothorax (deletion of the cost-diaphragmatic angle, partial or complete opacification of an hemithorax); pleural effusion (absence of breathing sounds, tactile vocal fremitus; homogeneous opacity with obliteration of the costophrenic angle); atelectasis (complete opacity of the hemithorax with mediastinal deviation).

Conclusion: The differential diagnosis of an opaque hemithorax is based on the position of the mediastinum and its volume, on a simple radiography. The three most likely entities in the patient of the clinical case were: hemothorax, pleural effusion and atelectasis. Complete radiopacity was associated with atelectasis, secondary to invasive adenocarcinoma.

Palabras Clave: Hemitórax opaco, derrame pleural, atelectasia, hemotórax, neumonectomía, adenocarcinoma.

Key Words: Opaque hemithorax, pleural effusion, atelectasis, hemothorax, pneumonectomy, adenocarcinoma.

\section{Introducción}

El hemitórax opaco, también conocido como velamiento pulmonar, es un hallazgo común entre los pacientes de salas de emergencias, y se define, según Marchiori, E., Hochhegger, B. y Zanetti, G. (2017) como una opacidad completa de uno de los hemitórax, en una radiografía simple de tórax (Marchori et al., 2017).

Opacidad es un término genérico inespecífico, que puede usarse en cualquier imagen que atenúa el haz de rayos $X$, haciendo que la imagen radiográfica sea más opaca o "blanca" (Wada, Rodrigues y Santos, 2019).El diagnóstico diferencial de esta imagen se basa en la posición del mediastino (específicamente de la tráquea, que da una mejor referencia), como se explica (Marchori et al., 2017):

-Volumen del hemitórax aumentado: el mediastino se desvía contralateral al lado afectado.

- Volumen del hemitórax disminuido: el mediastino se desvía hacia el lado afectado.

- Volumen del hemitórax normal: el mediastino no se desvía.

Cuando el volumen de un hemitórax opaco está aumentado, los diagnósticos diferenciales incluyen masas torácicas de gran tamaño (más frecuentes en niños), y derrame pleural masivo (lo cual constituye la causa más frecuente de este tipo de imagen); ambas pueden distinguirse utilizando Ultrasonido o una Tomografía Computarizada (TC) (Marchori et al., 2017). Un derrame pleural puede ser causado por una amplia variedad de etiologías, sin embargo, malignidad y tuberculosis son las más comunes encontradas en pacientes con derrames masivos (Vaidya, Vohra y Ghugare, 2017)

En el caso de un hemitórax opaco con reducción de volumen, pueden tomarse en cuenta diagnósticos como neumonectomía, agenesia, aplasia e hipoplasia pulmonar, y atelectasias; siendo las causas más frecuentes de ésta última, los tumores endobronquiales en adultos, y las obstrucciones bronquiales por cuerpos extraños en niños (Marchori et al., 2017).

Son pocos los casos de hipoplasia pulmonar, entidad caracterizada por una detención en el desarrollo pulmonar, habitualmente se diagnostica en infantes y tiene una alta mortalidad (Chumbi, Cubero, Jiménez, Ortega y Rodríguez, 2007),que pasan inadvertidos y se detectan en la edad adulta; sin embargo, en la mayoría, lo que orienta al diagnóstico son los hallazgos imagenológicos de un hemitórax opaco en una radiografía de tórax (Acosta et al., 2014) 
En la aplasia pulmonar, la presencia de un bronquio principal rudimentario que termina en saco ciego (reservorio de secreciones que se llenan a infectar), es lo que explica el cuadro clínico, generalmente pediátrico de infecciones respiratorias recurrentes, disnea, tos y estridor; pero se han reportado pacientes adultos asintomáticos. En los estudios radiológicos se aprecia una opacidad difusa de todo el hemitórax afectado, con desplazamiento mediastinal y elevación del hemidiafragma ipsilateral (SiegertOlivares, 2015)

Si no hay variación en el volumen, debe descartarse una neumonía grave que afecte la totalidad del parénquima pulmonar, si el paciente es pediátrico. $\mathrm{Si}$, por el contrario, se trata de un adulto, la causa más frecuente sería carcinoma bronquial, acompañado de atelectasias y derrame pleural (Marchori et al., 2017).

En el caso clínico que se presenta, la paciente es referida desde una clínica periférica, por cuadro de tos productiva de un mes de evolución, y hemoptisis de dos días de evolución asociada a disnea; en el servicio de emergencias (SEM) se le realiza una radiografía simple de tórax que revela una radioopacidad total del hemitorax izquierdo.

A continuación, se dan más detalles del caso clínico y la evolución de la paciente, también se explican además los diagnósticos diferenciales en los que los médicos tratantes pensaron al ver las imágenes médicas iniciales.

\section{Reporte del Caso}

Femenina de 70 años, vecina de Puriscal, Costa Rica. Hipertensa, en tratamiento con Amlodipina $5 \mathrm{mg} / \mathrm{d}$ V.O., y Enalapril $20 \mathrm{mg} / \mathrm{d}$ V.O. Niega uso de Aspirina. Sin otros antecedentes patológicos de importancia. Antecedente de cocinar con leña, niega tabaquismo y etilismo. Antecedente de Hernia Diafragmática Hiatal tipo III, resuelta por Toracotomía. G5P4C1A0. Hernia incisional abdominal.

Consulta a clínica periférica con historia de tos productiva de un mes de evolución, con hemoptisis de dos días de evolución, sensación febril, y disnea. Se encontraba hemodinámicamente estable, con saturación de Oxígeno de $84 \%$, sin alteración en el estado de consciencia, ni otros síntomas. En el examen físico se auscultan ruidos cardíacos rítmicos, sin soplos; los campos pulmonares limpios, con disminución de ruidos respiratorios en base izquierda. La paciente es referida al Hospital San Juan de Dios debido a que en la clínica periférica no se contaba con estudios radiológicos. La radiografía de tórax realizada en el SEM evidencia Radioopacidad total del hemitorax izquierdo.

Una TC de tórax con medio de contraste evidencia estructuras mediastinales con desplazamiento hacia la izquierda, secundario a atelectasia de pulmón izquierdo, persistiendo únicamente una pequeña zona ventilada en el LSI de aproximadamente $10 \%$ del volumen pulmonar. Bronquio principal izquierdo ocluido a $35 \mathrm{~mm}$ de la carina, englobado y ocupado por una masa sólida ovalada de $27 \times 27 \times 23 \mathrm{~mm}$, con densidad promedio de $60 \mathrm{UH}$. Adenopatía de $10 \times 10 \mathrm{~mm}$ en el nivel $2 \mathrm{~L}$, de $12 \times 12 \mathrm{~mm}$ en el nivel 5 , de $8 \times 16 \mathrm{~mm}$ en el $4 \mathrm{R}$, y de $29 \times 16 \mathrm{~mm}$ en el nivel 7. Pulmón derecho con sobredistensión compensatoria, trama vascular de calibre normal, micronódulos dispersos menores de $3 \mathrm{~mm}$, sin nódulos sospechosos derechos. Negativo por derrame pleural. Con impresión diagnóstica de "atelectasia obstructiva subtotal de pulmón izquierdo, secundaria a masa descrita".

Se realiza una broncoscopía donde se observa, en el bronquio principal izquierdo, a $4 \mathrm{~cm}$ de la carina, una lesión exofítica, blanquecina, friable, con vascularidad aumentada que obstruye el $100 \%$ de la luz bronquial; se toman muestras para su posterior análisis.

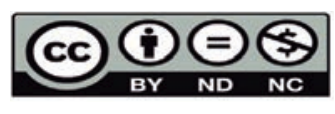


Una TC de abdomen y pelvis, como estudio de extensión, no evidencia enfermedad metastásica. A partir de estos resultados, los médicos tratantes consideran la resección pulmonar como primera opción de tratamiento.

Todo paciente en quien se considere una resección pulmonar como opción terapéutica, como en el presente caso, deber ser estudiado previamente mediante pruebas de funcionamiento pulmonar, para predecir el riesgo de complicaciones respiratorias y estimar la función pulmonar posoperatoria, así como predecir la probabilidad de que un paciente tolere adecuadamente la toracotomía y la posterior resección pulmonar. Debe evaluarse la función pulmonar estática (espirometría y DLCO), determinando de forma cuantitativa la cantidad de función pulmonar que podría perder con la cirugía (cálculo del FEV1).

Un FEV1 basal mayor del $60 \%$ del predicho se asocia a una morbilidad respiratoria del $12 \%$, un FEV1 menor del $30 \%$ del predicho, a una morbilidad de 43-60\% (Cid, León, Mejía, Torre y Gochicoa, 2018) La paciente del caso clínico tuvo un FEV1 de $79 \%$. Para las neumonectomías en específico, existe el método perfusorio, el cual aporta datos sobre la cantidad de V/Q que recibe cada pulmón, mide la fracción de la perfusión total a resecar, y evalúa así la posible función pulmonar posquirúrgica (Cid et al., 2018) La centellografía pulmonar $\mathrm{V} / \mathrm{Q}$ de la paciente permitió observar un $15,13 \%$ en pulmón izquierdo y $84,87 \%$ en pulmón derecho. Esta última se realiza como estudio complementario si FEV1 es $<80 \%$, que sería el valor más aceptado como seguro para el procedimiento quirúrgico (Mulholland et al., 2007)

Con los resultados anteriores, se procede a realizar una Neumonectomía, a cargo del departamento de Cirugía de Tórax. Se utiliza una toracotomía posterolateral izquierda para resecar pulmón y adenopatías sospechosas en estaciones 4, 6, 7, 9 y 10. El diagnóstico pos operatorio fue "Adenocarcinoma pulmonar izquierdo con compromiso de bronquio principal". La muestra se envía a patología quirúrgica, donde se confirma el diagnóstico con la biopsia, al reportarse un Adenocarcinoma invasor de 2,8 x1,9 cm, moderadamente diferenciado, con invasión linfovascular, infiltración neoplásica a ganglios linfáticos 5/18.

\section{Discusión}

Los tres diagnósticos diferenciales que se consideraron en la paciente del caso fueron hemotórax, derrame pleural y atelectasias, debido tanto a la presentación del cuadro clínico y la imagen radiológica, como a las características específicas de la paciente.

Éstos se explican a continuación:

\section{Hemotórax}

Puede definirse como la presencia de sangre en la cavidad pleural; sin embargo, debido a que un hematocrito igual o mayor al $5 \%$ en líquido pleural puede dar apariencia hemática, el diagnóstico se establece cuando el hematocrito en el líquido corresponde a un valor igual o mayor al $50 \%$ del valor en sangre periférica (Cortes, Morales, Figueroa, 2016). La radiografía de tórax de pie es el método diagnóstico inicial de elección; ésta brinda datos sugestivos como borramiento del ángulo costodiafragmático o una opacificación parcial o completa de un hemitórax. Radiografía de tórax de cúbito dorsal sólo se recomienda en los casos que no pueda realizarse la misma de pie (Campos y Vega, 2016).

El hemotórax es una de las complicaciones más comunes del trauma torácico. Además de esta causa, existen otras de origen no-traumático como las neoplasias torácicas y metástasis, tuberculosis, infecciones, malformaciones arteriovenosas pulmonares, sangrado iatrogénico consecuencia de toracocentesis o colocación de acceso venoso 
central (Camarillo-Reyes et al., 2019).

El hemotórax espontáneo ocurre con menor frecuencia, y suele generarse secundario a uso de anticoagulantes, anormalidades hematológicas como la hemofilia, tumores malignos, anormalidades vasculares congénitas pleuropulmonares, tromboembolismo con infarto pulmonar y neumotórax con adherencias pleurales (crónicos) (Cortes et al., 2016)

El trauma contuso menor, como el provocado por una caída desde su propia altura, puede ser particularmente peligroso en adultos mayores. $\mathrm{Al}$ respecto, Ota et al. (2018) explican que las caídas son actualmente el mecanismo de trauma torácico más común en individuos mayores de 70 años, lo cual resulta en una aumentada incidencia de fracturas costales y hospitalizaciones más largas que las asociadas con accidentes de tránsito. Mecanismos de trauma contuso menor pueden causar fracturas costales y hemotórax masivo.

El síndrome aórtico agudo, el cual incluye la disección aórtica y el hematoma intramural, puede ser la causa de un hemotórax, y a su vez, de un hemitórax opaco. Es importante considerarlo, ya que, si bien es más frecuente en hombres, la mayoría de los pacientes son adultos mayores en la octava década de vida, y se asocia frecuentemente a hipertensión arterial (Aouadi et al., 2016)

Las fracturas espontáneas de costillas causadas por tos, así como el hemotórax inducido por tos son poco comunes, pero se han reportado algunos casos, la mayoría asociados a episodios de tos severa. Esto puede ocurrir por dos mecanismos: el primero está relacionado a la fuerza aplicada, donde el estrés mecánico causa una deformación que, cuando excede el límite de elasticidad, resulta en una deformación in-elástica de la costilla esta clase de deformación se da en las partes más vulnerables de la costilla, ya sea en las uniones costocondrales, o en hueso osteoporótico, generalmente en el tercio medio de las costillas $5^{\circ}$ a la $10^{\circ}$. El segundo mecanismo está relacionado a las fuerzas musculares opuestas actuando sobre estos huesos; acciones opuestas de músculos intercostales y otros músculos respiratorios, pueden causar fracturas (Camarillo-Reyes et al., 2019).

\section{Derrame Pleural}

El derrame pleural se desarrolla cuando la producción de líquido aumenta en el espacio pleural, cuando disminuye la reabsorción del mismo, o una combinación de ambas (Skok, Hladnik, Grm y Crnjac, 2019) Muchas causas, tanto benignas como malignas, pueden llevar a la formación de líquido en exceso, siendo las más comunes la falla cardíaca, la neumonía y cáncer (Skok et al., 2019). Antes de dar tratamiento a un derrame pleural, se debe determinar si es un exudado o trasudado, según las características del líquido.

El examen físico puede orientar al diagnóstico de derrame pleural: la ausencia de ruidos respiratorios a la auscultación, matidez a la percusión y frémito vocal táctil son las características más comunes (Brogi et al., 2017). Sin embargo, el examen físico tiene menos sensibilidad y especificidad que el diagnóstico con imágenes, siendo la TC el gold standard.

Pueden utilizarse con este mismo fin, el ultrasonido torácico y la radiografía simple de tórax, siendo ésta última de fácil acceso en centros hospitalarios y capaz de mostrar derrames tan pequeños como $50 \mathrm{~mL}$ en una radiografía lateral en un paciente de pie; por el contrario, en una radiografía convencional posteroanterior (utilizadas en salas de shock y pacientes en cuidados intensivos), se requiere de al menos $200 \mathrm{~mL}$ para que el líquido sea visible, siendo esto una desventaja (Brogi et al., 2017). La imagen clásica en una radiografía simple es una opacidad homogénea ("blanca") con 
obliteración del ángulo costofrénico (Karkhanis y Joshi, 2012)

Si se determina que la causa del derrame pleural es cancerígena, se le llama derrame pleural por malignidad, y en un $95 \%$ es causado por metástasis en el espacio pleural; dos tercios de estos derrames son secundarios a cáncer de pulmón, mama y linfomas (Dixit et al., 2017); además entre el $70-77 \%$ de los casos están clasificados como Adenocarcinoma (Skok et al., 2019).

Los derrames pleurales por malignidad llevan a pérdida importante en la calidad de vida de los pacientes, debido a la disnea progresiva, tos seca, dolor torácico y reducción en la capacidad de realizar actividad física (Santos, Marques, Cruz, Monteiro y Fradinho, 2017) La mayoría de pacientes con este tipo de derrame aquejan disnea y su radiografía de tórax muestra derrame pleural de moderado a grande en $80 \%$ de los casos y derrame masivo en el $10 \%$ (Skok et al., 2019).

\section{Atelectasias}

Puede definirse como el colapso de una parte del pulmón y en consecuencia de sus alvéolos; este colapso implica una pérdida de volumen pulmonar, y puede ser total, segmentario o lobar, y determina la imposibilidad del intercambio gaseoso en las zonas comprometidas. La atelectasia es producida por una patología pulmonar o extrapulmonar (Tazza, Vásquez y Zapata, 2016) Las causas son múltiples, pero los escenarios clínicos más frecuentes son: carcinoma broncogénico, acúmulo de secreciones en la vía aérea, secuelas de tuberculosis y derrame pleural (Cortes, Che y Figueroa, 2016)

La radiografía de tórax es una herramienta muy útil para el diagnóstico, especialmente para descartar obstrucciones bronquiales centrales, como es el caso de una neoplasia endobronquial, siendo ésta la principal causa de colapso en adultos fumadores. No reconocer los signos que identifican una pérdida de volumen pulmonar, podría llevar a errores diagnósticos y retraso terapéutico. Por ejemplo, las opacidades lobulares pueden ser vistas como neumonías; podrían confundirse también las atelectasias con derrames, engrosamiento pleural o masas mediastínicas (Cortés y Martínez, 2014)

Al evaluar un paciente con sospecha de atelectasias, se deben considerar los signos radiológicos característicos: la opacidad aumentada de la zona, la reorientación de los vasos pulmonares, elevación del diafragma, desplazamiento del hilio y las fisuras, agrupamiento de las costillas, la hiperinsuflación compensatoria del pulmón sano, la desviación del mediastino y la rotación cardíaca (Reed, 2011). Una opacidad completa del hemitórax con desviación del mediastino, es la imagen clásica de una atelectasia completa de pulmón (Reed, 2011).

Las atelectasias son una condición común en pacientes con cáncer de pulmón, éstas se desarrollan usualmente por obstrucción endobronquial, y menos frecuente, por compresión causada por el tumor o derrame pleural (Bulbul et al., 2010).

\section{Conclusiones}

El diagnóstico diferencial de un hemitórax opaco en una radiografía simple, se basa en la posición del mediastino, y del volumen de éste, ya sea normal, aumentado o disminuido, según se aprecie en dicha imagen.

Existe gran cantidad de posibles diagnósticos, sin embargo, basados en la presentación inicial de la paciente del caso clínico, su sintomatología e imágenes médicas, las tres entidades más probables consideradas por los médicos tratantes fueron: hemotórax, derrame pleural y atelectasias. 
En la paciente del caso clínico, el hemitórax opaco era una radioopacidad completa asociada a una atelectasia obstructiva subtotal de pulmón izquierdo, secundaria a Adenocarcinoma invasor.

\section{REFERENCIAS BIBLIOGRÁFICAS}

Acosta, J., Arrué, A., Torres, B., Hierro, D., Tamayo, M., Álvarez, V. (2014) Hemitórax opaco. Revista Cubana de Medicina Militar; 43(1):122-128. Recuperado de: http://scielo.sld.cu/pdf/mil/v43n1/ mil14114.pdf

Aouadi, S., Sebai, A., Gharsalli, H., Zribi, H., Neji, H., Maâlej, S., Douik, L. (2016) Spontaneous Right Hemothorax in the Elderly. National Research Institute of Tuberculosis and Lung Disease, Iran. Tanaffos;15(1):57-60. Recuperado de: https:// www.ncbi.nlm.nih.gov/pmc/articles/PMC4937764/ pdf/Tanaffos-15-57.pdf

Brogi, E., Gargani, L., Bignami, E., Barbariol, F., Marra, A., Forfori, F., Vetrugno, L. (2017) Thoracic ultrasound for pleural effusion in the intensive care unit: a narrative review from diagnosis to treatment. Crit Care; 28;21(1):325. Recuperado de: https://www.ncbi.nlm.nih.gov/pmc/articles/ PMC5745967/pdf/13054_2017_Article_1897.pdf

Bulbul, Y., Eris, B., Orem, A., Gulsoy, A., Oztuna, F., Ozlu, T., Ozsu, S. (2010) Pulmonary atelectasis and survival in advanced non-small cell lung carcinoma. Ups J Med Sci. Aug; 115(3): 176-80.

Camarillo-Reyes, L. et al. (2019) Hemothorax induced by severe cough: An unusual presentation. SAGE Open Medical Case Reports; 7: 1-3

Campos, X., Vega, A. (2016) Hemotórax. Medicina Legal de Costa Rica - Edición Virtual; 33 (1): 25-34. Recuperado de: https://www.scielo.sa.cr/ $\mathrm{pdf} / \mathrm{mlcr} / \mathrm{v} 33 \mathrm{n} 1 / 1409-0015-\mathrm{mlcr}-33-01-00025$.pdf
Chumbi, R., Cubero, N., Jiménez, L., Ortega, A., Rodríguez, M. (2007) Hipoplasia pulmonar en el adulto. Rev Patol Respir; 10(4): 194-196

Cid, S., León, P., Mejía, R., Torre, L., Gochicoa, L. (2018) Evaluación de la función respiratoria en pacientes que van a ser sometidos a cirugía de resección pulmonar. Neumol. cir. torax; 77(1): 3846. Recuperado de: http://www.scielo.org.mx/pdf/ nct/v77n1/0028-3746-nct-77-01-38.pdf

Cortes, A., Che, J., Figueroa, E. (2016) Atelectasia lobar. Manifestación infrecuente de traqueobroncomalacia en adultos. Rev. biomédica. Abr; 27(1): 25-30. Recuperado de: http://www.scielo. org.mx/pdf/revbiomed/v27n1/2007-8447-revbiomed-27-01-25.pdf

Cortés, A., Martínez, M. (2014) Manifestaciones radiográficas de las atelectasias pulmonares lobares en la radiografía de tórax y su correlación con la tomografía computarizada. Radiología; 56(3): 257-267

Cortes, A., Morales, C., Figueroa, E. (2016) Hemotórax: etiología, diagnóstico, tratamiento y complicaciones. Rev Biomed; 27:119-126 Recuperado de: http://www.scielo.org.mx/pdf/revbiomed/v27n3/2007-8447-revbiomed-27-03-119.pdf

Dixit, R., Agarwal, K., Gokhroo, A., Patil, C., Meena, M., Shah, N., Arora P. (2017) Diagnosis and management options in malignant pleural effusions. Lung India. Mar-Apr; 34(2): 160-166.

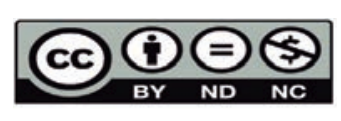


Karkhanis, V., Joshi, J. (2012) Pleural effusion: diagnosis, treatment, and management. Open Access Emerg Med. 22; 4:31-52.

Marchiori, E., Hochhegger, B., Zanetti, G. (2017) Opaque hemithorax. J Bras Pneumol. 43(3):161. Recuperado de: http://www.scielo.br/pdf/jbpneu/ v43n3/1806-3713-jbpneu-43-03-00161.pdf

Mulholland, M., Lillemoe, K., Doherty, G., Upchurch, G., Alam, H. Pawlik, T. (2007) Greenfield's Surgery: Scientific Principles \& Practice. Sixth Edition. Philadelphia, U.S.A. Wolters Kluwer. Pág. 303

Ota, K., Fumimoto, S., lida, R., Kataoka, T., Ota, K., Taniguchi, K., Hanaoka, N., Takasu, A. (2018) Massive hemothorax due to two bleeding sources with minor injury mechanism: a case report. J Med Case Reports; 12(1): 291. Recuperado de: https:// jmedicalcasereports.biomedcentral.com/track/ pdf/10.1186/s13256-018-1813-x

Reed, J. Chest Radiology. Sexta Edición. Philadelphia, USA. Elsevier. 2011

Santos, P., Marques, M., Cruz, C., Monteiro, H., Fradinho, F. (2017) Predictors of talc slurry pleurodesis success in patients with malignant pleural effusions. Rev Port Pneumol; 23(4): 216-220

Siegert-Olivares, A., Penchyna, J., Balderas, L., Jaramillo, C., Saenz, J., Bechara, J. (2015) Aplasia pulmonar: a propósito de dos casos. Bol Med Hosp Infant Mex; 72 (1): 66-70. Recuperado de: https:// www.researchgate.net/publication/277969879_ Aplasia_pulmonar_a_proposito_de_dos_casos Skok, K., Hladnik, G., Grm, A., Crnjac, A. (2019) Malignant Pleural Effusion and Its Current Management: A Review. Medicina (Kaunas); 55(8):490.
Tazza, F., Vásquez, R., Zapata, J. (2016) Atelectasia aguda súbita pulmonar izquierda por linfoma no Hodgkin de mediastino anterior y superior. Acta méd. Peruana. Abr; 33(2): 150-154. Recuperado de: http://www.scielo.org.pe/pdf/amp/v33n2/ a11v33n2.pdf

Vaidya, V., Vohra, P., Ghugare, B. (2017) Opaque hemithorax: Clinical, histological and radiological assessment of 30 cases at a tertiary care hospitala preliminary study. West Afr J Radiol; 24:34-7

Wada, D., Rodrigues, J., Santos, M. (2019) Semiologia radiológica e terminologia da radiografia de tórax. Medicina (Ribeirao Preto Online) 52(supl1.): 31-43. Recuperado de: https://www.revistas.usp. $\mathrm{br} / \mathrm{rmrp} /$ article/view/154795 